the trans-carotid group, but not statistically significant. There was no difference between groups regarding the 90-days mRS outcome (3.88 vs $3.87, \mathrm{p}=0.95)$. Two patients in transcarotid group suffered an iatrogenic cervical ICA dissection and a small pseudoaneurysm $1 \times 5 \mathrm{~mm}$ without incident. Two patients also developed neck hematomas that were managed with conservative manual compression.

Conclusion A trans-carotid approach is a relatively safe alternative to transfemoral approach in MT, and may be considered in patients with a type 3 aortic arch, cervical vasculature with $>1$ tortuous segment ( $>90$ degrees), and/or an angular tortuosity summing $>360$ degrees from the aortic arch to the carotid bifurcation. These patients were more likely to be older and have a history of previous stroke/TIA.

Disclosures M. Aly: None. R. Abdalla: None. M. Hurley: None. A. Shaibani: None. S. Ansari: None.

\section{E-101 LOW DOSE EPTIFIBATIDE IN THE MANAGEMENT OF TANDEM OCCLUSION}

${ }^{1} \mathrm{~A}$ Jost ${ }^{*},{ }^{2} \mathrm{C}$ Roels, ${ }^{3} \mathrm{M}$ Brown, ${ }^{4} \mathrm{R}$ Janjua, ${ }^{3} \mathrm{D}$ Heck. ${ }^{1}$ School of Medicine, Wake Forest University, Winston Salem, NC; ${ }^{2}$ Pharmacy, Forsyth Medical Center, Winston Salem, NC; ${ }^{3}$ Radiology, Forsyth Medical Center, Winston Salem, NC; ${ }^{4}$ Neurosurgery, Forsyth Medical Center, Winston Salem, NC

10.1136/neurintsurg-2020-SNIS.134

Introduction Tandem intracranial/extracranial occlusion presents a challenge due to the coincident risks of symptomatic intracranial hemorrhage $(\mathrm{SICH})$ and carotid re-occlusion. Various anti-thrombotic regimens have been reported with mixed results for SICH, but infrequent analysis of cervical carotid patency. We present a single center, retrospective analysis of tandem occlusion patients treated with low dose eptifibatide, with emphasis on rates of hemorrhage and rigorous reporting of cervical carotid artery patency. The work is unique due to the completeness of vessel imaging follow-up.

Materials and Methods $\mathrm{N}=58$ patients were administered a low dose perioperative eptifibatide regimen $(135 \mathrm{mcg} / \mathrm{kg}$ bolus, $1 \mathrm{mcg} / \mathrm{kg} / \mathrm{min}$ infusion) during intracranial thrombectomy and extracranial carotid angioplasty \pm stent placement. A prospective database was reviewed retrospectively for patient characteristics, clinical and imaging outcomes. Determination of SICH was per the SITS-MOST definition. Management of the intracranial occlusion by direct aspiration (ADAPT) or combination therapy (CAPTIVE) was per physician choice, as was management of the extracranial lesion with stenting or angioplasty alone. Patients were converted to dual oral anti-platelet therapy with aspirin and clopidogrel or ticagrelor if the 24 hour ultrasound and CT demonstrated carotid patency and no significant hemorrhage.

Results The average age and NIHSS were 64 and 16, respectively, with tPA use in $43 \%$ of cases. ASPECTS scores were $8-10$ in $\mathrm{N}=47$ (81\%) and $5-7$ in $\mathrm{N}=11$ (19\%). $\mathrm{N}=38$ patients had stenting acutely, while $\mathrm{N}=20$ had angioplasty alone, 6 of whom had delayed endarterectomy or stenting within the first week. All patients had brain imaging within 24-36 hours. Any evidence of intracranial hemorrhage was present in $\mathrm{n}=18$ (29\%), mostly petechial or small subarachnoid hemorrhage, with only one case of SICH (2\%). Technical success (TICI 2B or 3) was achieved in $\mathrm{N}=56$ (96\%). 90 day MRS was documented by phone or in person in $57 / 58$ patients (one patient lost to follow up). 90 day MRS of 0-2 was achieved in $\mathrm{N}=42(72 \%)$, with MRS of 6 in $\mathrm{N}=5$ (9\%).
All patients had duplex within 36 hours. There were $\mathrm{N}=4$ (7\%) acute re-occlusions, all in patients originally treated with stenting. $\mathrm{N}=49$ patients had carotid imaging at 30-60 days (5 deceased, 3 not done, 1 lost to follow up), with an additional $\mathrm{N}=3$ re-occlusions (6\%), two of whom had stents and one angioplasty alone. Carotid artery patency at 30-60 days for patients imaged was $86 \%(42 / 49)$. No acute or delayed carotid occlusion was associated with clinical decline, and 5/7 (71\%) had 90 day MRS of 0-2.

Conclusion Within this cohort, low dose eptifibatide in the tandem occlusion population seemed to be safe with a low incidence of SICH. Cervical carotid artery re-occlusions did occur with an overall 30-60 day patency of 86\%. Re-occlusion was not associated with clinical decline in any patient. In the absence of a control arm, it is unknown whether cervical carotid patency is improved with low dose eptifibatide compared to any other regimen. All conclusions are limited by the small sample size, retrospective nature of the study, and lack of core lab adjudication of outcomes.

Disclosures A. Jost: None. C. Roels: None. M. Brown: None. R. Janjua: None. D. Heck: 2; C; Stryker.

\section{E-102 ECONOMIC AND CLINICAL MODEL FOR DIRECT TRANSFER TO ANGIOSUITE PROTOCOL DEVELOPMENT}

${ }^{1} M$ Requena*, ${ }^{2} V$ Seguel, ${ }^{1} \mathrm{M}$ Olivé-Gadea, ${ }^{1} \mathrm{~A}$ García-Tornel, ${ }^{1} \mathrm{~A}$ Vilaseca, ${ }^{1} \mathrm{~N}$ RodríguezVillatoro, 'M Deck, 'J Juega, 'S Boned, 'M Muchada, ${ }^{1} \mathrm{C}$ Piñana, ${ }^{1} \mathrm{~J}$ Pagola, ${ }^{1} \mathrm{D}$ RodríguezLuna, ${ }^{1} \mathrm{D}$ Hernández, ${ }^{1} \mathrm{M}$ Rubiera, ${ }^{1} \mathrm{C}$ Molina, ${ }^{1} \mathrm{~A}$ Tomasello, ${ }^{1} \mathrm{M}$ Ribo. ${ }^{1}$ Stroke Unit, Vall $d^{\prime}$ Hebron University Hospital, Barcelona, SPAIN; ${ }^{2}$ Neurovascular Health Economics and Reimbursement, Medtronic UK, Watford, UK

\subsection{6/neurintsurg-2020-SNIS. 135}

Introduction Direct transfer to angiosuite (DTAS) has consistently shown to be effective and safe shortening in-hospital workflows and encouraging long-term outcome benefits. In order to generalize DTAS an organizational and manpower effort is necessary. We aim to perform a cost-effective analysis of the implementation of a new angiography suite primarily dedicated to DTAS of stroke patients that will allow generalization of this pathway.

Material and Methods 61 patients who underwent endovascular treatment (EVT) following DTAS were matched for baseline variables to 117 patients who underwent conventional 
imaging protocol before EVT. An economic model based on current data was developed to assess the short and long term clinical and economic implications. The DTAS development scenario estimates a gradual 20\% increase of DTAS rate for 4 years followed by a stable $80 \%$ rate of DTAS. Initial investment and additional organizational costs were included: $4 \mathrm{M}$ $€$. A cost-effective study compared the DTAS development scenario (SC1) to a scenario with no organizational changes (SC2) over 10 years.

Results The 10 year model included 1775 EVT patients in each scenario: SC1 68\% DTAS Vs SC2 0\% DTAS. SC1 would be associated with better functional independence rates (mRS $0-2$ : $45.8 \%$ versus $40.2 \% \mathrm{p}=0.04$ ) and a quality-adjusted lifeyears gain of 0.12 per patient. Despite the additional investment, SC1 development was associated with an estimated $15.1 \%$ reduction $(26.25 \mathrm{M} €)$ of total costs $(173.749 \mathrm{M} €)$. Cost saving was mainly due to long-term associated costs related with patient disability ( $€ 26.4$ million).

Conclusions Our economic model predicts that the development of a DTAS program is cost effective.

Disclosures M. Requena: None. V. Seguel: None. M. OlivéGadea: None. Á. García-Tornel: None. A. Vilaseca: None. N. Rodríguez-Villatoro: None. M. Deck: None. J. Juega: None. S. Boned: None. M. Muchada: None. C. Piñana: None. J. Pagola: None. D. Rodríguez-Luna: None. D. Hernández: None. M. Rubiera: None. C. Molina: None. A. Tomasello: None. M. Ribo: 2; C; Medtronic, Stryker, Johnson and Johnson, Anaconda Biomed, Apta Targets.

\section{E-103 DESIGN AND EVALUATION OF AN XMR-VISIBLE CATHETER FOR STROKE APPLICATIONS}

'B Kilbride*, C Jordan, 941071, '2A Chu, '2D Barry, T Moore, 94107', A Martin, 94107', M Wilson, 94107', S Hetts, 94107'. 'Radiology and Biomedical Imaging, University of California San Francisco, San Francisco, CA; ${ }^{2}$ Penumbra Inc., Alameda, CA

\subsection{6/neurintsurg-2020-SNIS.136}

Introduction Acute ischemic stroke can be treated effectively under X-ray guidance, but eligibility for such treatment is limited by small time windows following symptom onset.
Treatment windows could be extended with MRI information since diffusion weighted imaging and perfusion imaging are the gold standard in determining if brain tissue is still salvageable by reperfusion therapy. Lack of safe tooling in such unique environments has limited catheter-based interventions as commercial devices use metallic reinforcement, introducing risk of RF-induced heating. We present a polymer-based catheter with a passive ink-based marker to create low-profile, MR-visible markers that can also be visualized under X-ray. Prototype feasibility was demonstrated in vitro and mechanically compared to a commercial guide catheter.

Methods Prototypes were built in a catheter fabrication facility (Penumbra, Inc., Alameda, CA). Polymer-based filament was wound onto an inner plastic liner. Four circumferential passive marker bands were painted $2 \mathrm{~cm}$ from the distal tip. Markers were an epoxy-based radiopaque ink (Creative Materials, Ayer, MA) doped with iron(III) oxide $\left(\mathrm{Fe}_{2} \mathrm{O}_{3}\right)$ nanoparticles of $20-40 \mathrm{~nm}$ diameter (Alfa Aesar, Tewksbury, MA). The resulting subassembly was laminated with a multi-durometer thermoplastic jacket. MRI experiments were performed in a clinical hybrid interventional XMR suite. The catheter was submerged in water oriented parallel to $\mathrm{B}_{0}$ on a $1.5 \mathrm{~T}$ MRI scanner (Achieva, Philips, Best, The Netherlands). A gradient echo (GRE) and fast spin echo (TSE) were acquired. X-ray images were captured using a Cios Alpha portable C-arm (Siemens Healthineers, AG, Forccheim, Germany). Kink resistance was quantified with a benchtop fixture against a commercial guide catheter of similar dimensions.

Results The final device had outer and inner diameters of $2.36 \mathrm{~mm}$ and $1.83 \mathrm{~mm}$, respectively, and a wall thickness of $0.265 \mathrm{~mm}$. Negative contrast signal caused by the markers showed good tracking characteristics in the GRE and TSE sequences (figure 1). Radiopaque markers were visualized under X-ray in the water phantom. Prototype and commercial catheter distal kink radii were $7.5 \mathrm{~mm}$ and $11.0 \mathrm{~mm}$, respectively. The prototype exhibited a larger wall thickness than the commercial device.

Conclusions MR and X-ray images demonstrated that the passive markers possessed good negative contrast signal at $1.5 \mathrm{~T}$
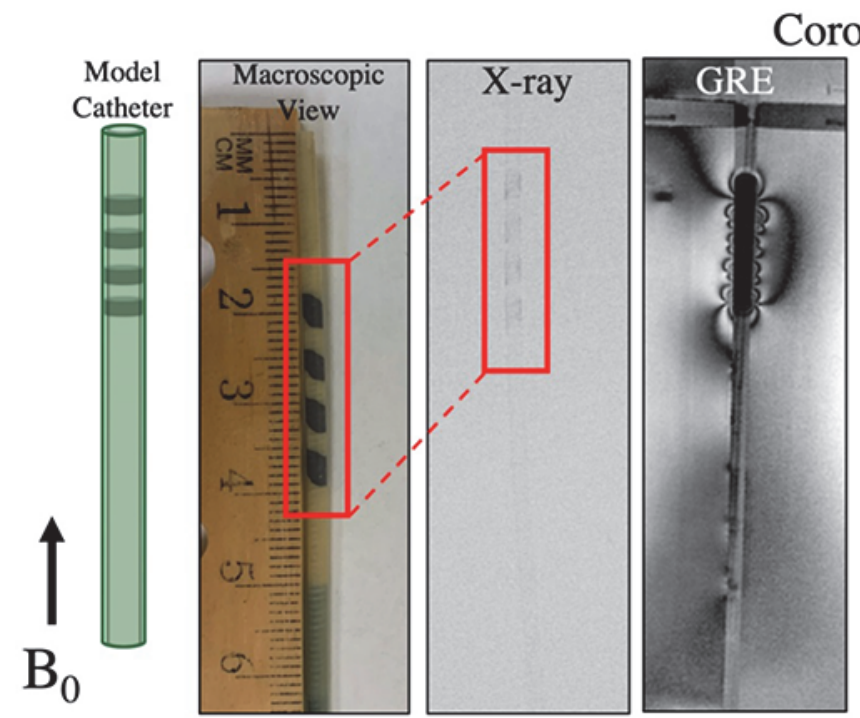

Coronal

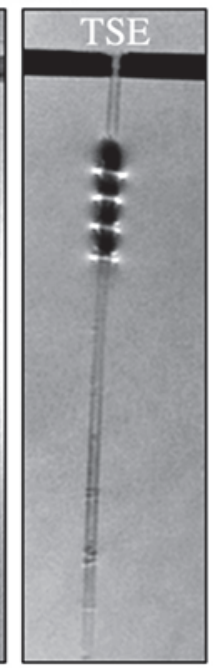

Sagittal

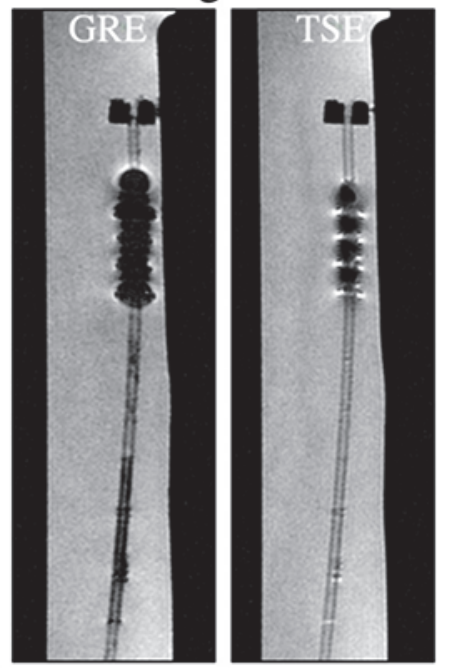

Abstract E-103 Figure 1 Visualization of prototype under X-ray and MR 